Revue musicale OICRM

\title{
Louise Campbell. Une médiatrice dans l’être
}

\section{Fanny Bertomeu}

Volume 7, numéro 2, 2020

URI : https://id.erudit.org/iderudit/1072417ar

DOI : https://doi.org/10.7202/1072417ar

Aller au sommaire du numéro

\section{Éditeur(s)}

Observatoire interdisciplinaire de création et recherche en musique (OICRM)

\section{ISSN}

2368-7061 (numérique)

Découvrir la revue

\section{Citer ce document}

Bertomeu, F. (2020). Louise Campbell. Une médiatrice dans l'être. Revue musicale OICRM, 7(2), 92-100. https://doi.org/10.7202/1072417ar

\section{Résumé de l'article}

Musicienne et clarinettiste de formation, Louise Campbell est aujourd'hui médiatrice de la musique. Basée à Montréal, elle exerce sa profession au Québec comme au Canada anglophone, à travers plusieurs structures et pour des publics variés (scolaires, professionnels, personnes en situation de handicap...). Dans cet entretien, Louise Campbell raconte son expérience de médiatrice, sa (non-)formation à cette profession ainsi que les aspects de sa pratique. Avec l'objet musical et la création au coeur de ses ateliers, Louise Campbell cherche toujours à établir une relation d'échange " horizontale " avec chacune des personnes qu'elle rencontre. Médiatrice dans l'être, Louise Campbell est aussi une médiatrice de l'être. 


\title{
Louise Campbell. Une médiatrice dans l'être
}

\author{
Fanny Bertomeu
}

\begin{abstract}
Résumé
Musicienne et clarinettiste de formation, Louise Campbell est aujourd'hui médiatrice de la musique. Basée à Montréal, elle exerce sa profession au Québec comme au Canada anglophone, à travers plusieurs structures et pour des publics variés (scolaires, professionnels, personnes en situation de handicap...). Dans cet entretien, Louise Campbell raconte son expérience de médiatrice, sa (non-) formation à cette profession ainsi que les aspects de sa pratique. Avec l'objet musical et la création au cœur de ses ateliers, Louise Campbell cherche toujours à établir une relation d'échange " horizontale " avec chacune des personnes qu'elle rencontre. Médiatrice dans l'être, Louise Campbell est aussi une médiatrice de l'être.
\end{abstract}

Mots clés : Louise Campbell ; création ; médiation de la musique ; partage ; relation.

\begin{abstract}
Formed as a musician and a clarinetist, Louise Campbell is now a music mediator. Based in Montreal, she practices her profession through several structures and for a wide variety of audiences (schoolchildren, professionals, people with disabilities ...) all around Canada, but mainly in Quebec. In this interview, Louise Campbell recounts her experience as a mediator, her (non)-formation in this profession, as well as many aspects in her practice. With the musical object and the creation at the heart of her workshops, Louise Campbell always seeks to establish a "horizontal" exchange relationship with each person she meets.
\end{abstract}

Keywords: Louise Campbell; creation; music mediation; sharing; relationship. 


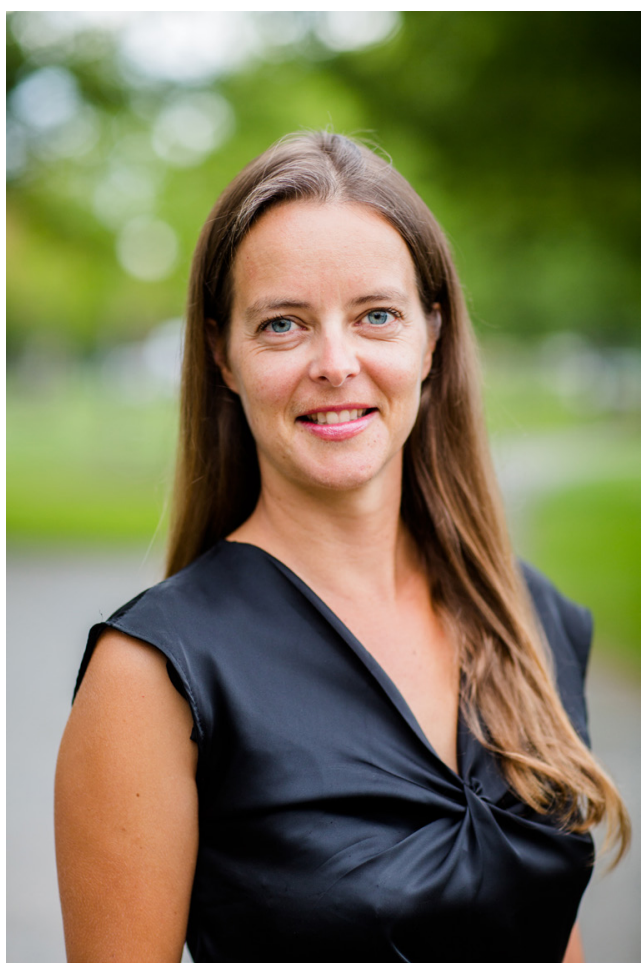

Louise Campbell. Photo :

Vivian Doan Photography, 2017.
Louise Campbell est clarinettiste, compositrice et médiatrice de la musique. Bien qu'elle vive et travaille à Montréal, elle est régulièrement sollicitée pour intervenir à divers endroits du Québec et du Canada (de Montréal aux Îles-de-la-Madeleine en passant par Ottawa). Équipée de sa clarinette, forte de son expérience de terrain et de son bagage artistique, elle part à la rencontre de publics toujours différents avec lesquels elle crée des moments de découverte et de partage autour de la musique.

Louise Campbell travaille avec des structures et des organismes de production et de diffusion musicale variés, francophones et anglophones, tels quel'Orchestre symphonique de Montréal, Bradyworks et Innovation en concert. Elle est aussi souvent engagée dans le domaine scolaire notamment par l'intermédiaire du programme La culture à l'école mis en place par le ministère de l'Éducation ainsi que 1'ELAN ACE Initiative.

En parallèle aux actions de médiation qu'elle réalise, la médiatrice se passionne pour les questions de formation et de transmission de sa pratique. C'est ainsi qu'elle a créé un blogue qu'elle alimente régulièrement en articles destinés à accompagner des professeur·e·s d'école qui ne disposeraient pas nécessairement d'outils pour mener à bien des activités de médiation de la musique avec leurs élèves.

Lors de notre rencontre en août 2019, Louise Campbell collaborait avec le Réseau canadien des musiques nouvelles à la création de ressources en ligne présentant les «bonnes pratiques » à mettre en place pour faire de la musique participative et créative avec les gens.

En définitive, Louise Campbell est engagée activement sur plusieurs fronts pour rendre la musique accessible et épanouissante pour le plus grand nombre. Aux vues de la teneur de ses propos recueillis lors de notre entretien, il ressort deux axes de réflexion dominants : d'une part, la nécessité pour la médiatrice d'installer une relation de réciprocité et de partage avec son public ; d'autre part, la difficulté qu'elle rencontre à identifier les contours et les enjeux d'une profession de " médiatrice » alors qu'elle pratique ce mode d'intervention artistique et social de manière intuitive. 
À l'instar de Bruno Péquignot ${ }^{1}$ et de Jean-Pierre Saez ${ }^{2}$, Louise Campbell souligne l'incommensurabilité de la question relative à la définition de la médiation. Notre entretien l'amène pourtant progressivement à cerner les contours de cette pratique à partir de la nécessité d'établir et nourrir une relation avec des publics toujours différents. Dans sa définition de la médiation, la musique joue alors à la fois le rôle de prétexte et d'objet des rencontres. Chez elle, la musique devient l'élément déclencheur de la relation. Une fois ce contact établi, elle fait vivre une expérience musicale à des publics qui étaient parfois très éloignés de son vocabulaire musical ${ }^{3}$. La médiatrice-musicienne cherche ainsi à créer une relation unique avec chacune des individualités dont la somme constitue les publics de ses activités.

Pour Louise Campbell, la médiation de la musique consiste alors à savoir créer cette dynamique homogène par une approche à la fois intime et collective, toujours unique. Elle l'affirme : l'important n'est pas de considérer les compétences et les connaissances des participant $\cdot e \cdot s$ (même si elle peut volontiers s'en servir) ni même le « produit final » d'une activité de médiation, mais bien de parvenir à la possibilité d'une rencontre, d'une relation.

Fanny Bertomeu : Qu'est-ce que la médiation de la musique pour toi ?

Louise Campbell : C'est une grande question! La médiation de la musique, pour moi, c'est juste une façon de faire en sorte que tout le monde, peu importe son background ou sa formation (ou son absence de formation), ait une porte d'accès à la musique. Faire de la médiation de la musique, c'est trouver des moyens pour que les gens vivent une belle expérience à travers la musique. Pas forcément pour mieux comprendre la musique de manière absolue et universelle, mais plutôt pour mieux comprendre la musique en mobilisant ses acquis. Donc peu importe que les participants soient très jeunes ou plus âgés, ils vont s'amuser avec leur façon de faire, avec leurs connaissances de vie, de métier et tout leur bagage de vie. La chose importante en médiation, c'est de trouver des liens avec la personne et de trouver la façon de la captiver avec la musique. [...] Mais ça dépend aussi du public à qui je parle. Il y a des gens qui viennent d'horizons ou de métiers vraiment très différents des miens ! Si je peux aussi apprendre d'eux, c'est tant mieux! Par exemple, si je parle avec un e architecte, on va aborder les choses différemment que si je parle avec un homme d'affaire. Peut-être que l'architecte, qui connaît mieux l'acoustique que moi, pourrait être intéressé e de savoir que les pièces de Haydn étaient jouées dans des endroits différents et que ça avait un impact sur la composition et l'interprétation. Donc c'est vraiment l'idée d'un

1 Bruno Péquignot (2007), «Sociologie et médiation culturelle », L'Observatoire, vol. 32, n 2, p. 3-7.

2 Jean-Pierre Saez (2018), « Les paradoxes de la médiation culturelle », L'Observatoire, vol. 51, n 1, p. $1-2$.

3 Louise Campbell est particulièrement active dans le champ des musique actuelles (au sens québécois de l'intitulé) et expérimentales. Elle se produit notamment avec In Extensio, son ensemble avec lequel elle cocrée et commande de nouvelles œuvres. Louise est également influencée par les musiques jazz (David Baker). 
partage avec des individualités singulières, mais intégrées dans un groupe. Souvent aussi, les gens se font des idées de la musique qui ne sont pas forcément celles de la réalité, alors j'essaye de déconstruire ça doucement pour que ces gens comprennent qu'eux, ils peuvent faire la musique eux-mêmes. C'est pour cela aussi que je n'ai pas seulement fait de la musique participative, mais de la musique à la fois participative et créative pour faire en sorte que ce soient les gens qui créent leur propre musique. Souvent, ils vont trouver que c'est à la fois plus facile et plus difficile que ce qu'ils imaginaient.

Alors que la question de la participation des publics est au cour de la réflexion sur la spécificité de la médiation culturelle ${ }^{4}$, Louise Campbell va encore plus loin. Dépassant ce qu'on qualifie de paradigme participatif, la médiatrice engage les participant $\cdot e \cdot s$ à faire acte de création. Elle mise ainsi sur une médiation qui engage pleinement son public dans des productions sonores qui permettent l'expression sensible de chacun'e. Les participant·e's sont actif·ve's voire même proactif·ve's lorsque, dans une cocréation, ils ou elles sont à l'initiative des choix qui permettront la faire émerger collectivement une réalisation artistique. Cette posture de médiatrice s'accompagne d'une réflexion sur les compétences propres au métier de médiateur-trice. Pour Louise Campbell, s'adapter à ses publics et avoir le sens du rebond sont sans conteste des qualités nécessaires dans ce type d'activité.

L. c. : Pour moi, il y a toujours une grande partie de l'atelier qui est participatif. Parce que je trouve que quand on fait de la musique, on la comprend différemment lorsqu'on l'écoute par la suite. Donc même si je fais la médiation et que je suis engagée par un organisme pour des gens qui vont aller assister à un concert, je vais toujours faire quelque chose de participatif qui est en lien avec le concert, avec les œuvres qu'ils vont entendre. L'idée est de faire en sorte qu'ils partagent une expérience créative en lien avec la musique qui va être jouée par la suite. [...] J'ai plusieurs axes et j'utilise le bon outil pour le bon job. Par exemple, si j'ai besoin d'aller faire de la médiation avec des personnes âgées et que je sais qu'il n'y a pas de piano dans les locaux, ni de microphone et que ma voix ne va pas porter très loin pour ces personnes qui commencent à perdre un peu l'audition, là je vais amener ma clarinette parce que je peux jouer bien plus fort avec ma clarinette qu'avec ma voix. Ça sera plus facile de commencer à faire un signal avec ma clarinette. Si j'anime un atelier autour de la musique de film avec des adolescents, peut être que je vais amener ma clarinette. Peut-être que je vais seulement l'utiliser 30 secondes et que ça ne va pas être très utile. Dans ce cas-ci, je vais aussi amener mon ordinateur pour avoir accès à de la musique via des plateformes de contenus. On peut aller chercher des choses sur YouTube que les ados ont envie d'écouter pour créer une relation avec eux et que cela débouche sur

4 Voir : Marion Carrel (2017), «Injonction participative ou empowerment ? Les enjeux de la participation ", Vie sociale vol. 3, no 19, p. 27-34, https://www.cairn.info/revue-vie-sociale-2017-3-page-27.htm, consulté le 23 avril 2020 ; Caroline Urbain et Danielle Bouder-Pailler (dir.) (2016), Participation et médiation(s). Nouveaux regards pour de nouveaux enjeux, Paris, L'Harmattan. 
une curiosité, un désir, un intérêt. Quelquefois je vais aussi amener ma clarinette avec des pédales. Ça, je le fais souvent dans des écoles pour les harmonies, c'est une façon pour moi de faire une démonstration : « OK, on a fait ces activités-là, maintenant voici une façon de faire toute une pièce qui pourrait ressembler à quelque chose pour toute une harmonie. »C'est une manière de faire semblant que je suis un band!

J'utilise les choses dont j'ai besoin. Je dirais que j'ai des façons de faire qui sont assez personnelles. Quelqu'un d'autre qui fait de la médiation va aussi utiliser ses propres façons de faire, qui vont lui être singulières. [...] J'ai toujours une structure générale et des façons de faire qui, je le sais grâce à mon expérience, vont fonctionner. À partir de là, oui, je peux improviser avec du monde, avec les gens qui sont devant moi. Donc même si je commence avec une petite idée de départ, les gens présents vont parfois l'amener vers une direction complètement autre. Je vais donc suivre leurs idées et aussi leur donner d'autres façons de faire. Mais parfois une petite idée peut prendre cinq voies complètement différentes avec cinq groupes complètement différents.

F. в. : Quelle est la place de l'imprévu et de l'improvisation dans tes ateliers?

L. c. : Je peux raconter une histoire d'un atelier qui m'avait beaucoup surprise! J'étais engagée pour faire des ateliers, c'était un engagement dans le centre C.A.R.E. qui est le Centre d'Activités Récréatives et Éducatives. C'est un programme de journée pour les adultes qui vivent avec des handicaps divers. C'était la première fois que je faisais un atelier de médiation de la musique de ce genre-là, donc j'étais un peu nerveuse. Je n'étais pas sûre de moi, je n'avais pas prévu de choses spécifiques comme : « OK, on va commencer avec ça, ensuite je sais que j'ai cinq options différentes. »C'était plutôt du genre : «OK, faut juste que j'essaye des choses. » Donc j'y vais et je découvre petit à petit ce qu'il est possible de faire sur place. Je suis donc allée là-bas, et j'ai d'abord participé à une activité qu'une autre personne était en train d'animer. J'observais comment ça allait avec les gens dans la salle. C'était très confortable, c'étaient des gens qui se connaissaient très bien, qui rigolaient beaucoup. C'était vraiment facile comme environnement, j'étais très à l'aise avec ça. Puis j'ai commencé à observer comment des gens interagissaient et communiquaient entre eux. Il y avait des gens qui parlaient avec un ordinateur, d'autres avec un système de communication avec les yeux. C'est un système qui accorde des chiffres avec la direction des yeux. C'était donc possible pour moi de communiquer avec cette personne qui utilisait Bliss ${ }^{5}$. Si cette personne communiquait 1-0-0-1-1 avec ses yeux, ça correspondait à une combinaison particulière dans une charte. Même sij'ai été hyper lente à comprendre, c'était possible de communiquer! Tous ces gens avaient des habitudes différentes. Peu importait si c'était quelqu'un qui pouvait beaucoup bouger, de très grand et avec beaucoup de force ou quelqu'un qui pouvait seulement bouger la tête avec une force très faible... On avait devant nous des objets tirés de leur bac de recyclage et on construisait des

$5 \quad$ Système de communication alternative non-verbale, le Bliss permet à des personnes présentant des difficultés à parler à s'exprimer grâce à un ensemble de symboles visuels simples. Pour en savoir plus, voir : https://www.handirect.fr/communication-alternative-celia/, consulté le 12 mai 2020. 
instruments de musique avec tout ce qu'on avait sous la main. Ça, c'était le concept de l'atelier. Je pense que ces gens-là n'avaient pas fait leur recyclage depuis des mois ! Il y avait plein d'affaires. Entre autres, il y avait plein de petites canettes de thé qui sonnaient super bien quand on les frappe avec une baguette. C'est la première fois que je voyais autant de petites canettes de thé ! On avait donc construit plusieurs instruments, comme certains qu'on pouvait par exemple porter sur le poignet... On avait aussi construit un instrument avec le système de communication Bliss, avec les canettes. Il y avait aussi un monsieur qui était là, très bon avec ses mains, qui adorait ça construire. Je lui avais demandé de construire un petit cadre. Quant à moi, je suis plutôt bonne avec les nœuds. À nous deux, on avait mis en place une certaine façon de suspendre les canettes de thé comme dans le système de communication Bliss. De cette manière, la personne qui communiquait avec Bliss pouvait me donner des notes de musique à jouer. À la fin, c'est même elle qui dirigeait. Tout le monde avait son propre instrument et c'était elle qui disait aux autres quand jouer et quand arrêter. C'était un moyen de faire en sorte que tout le monde dans la salle puisse contribuer selon ses propres capacités.

Louise Campbell cherche donc, en fonction de ses préférences, à capter l'attention de son auditoire, à " entrer en relation » avec les participants afin de faire germer le désir de l'écoute musicale. Selon les contextes, la médiatrice utilise des processus créatifs faisant intervenir de multiples facteurs d'adhésion tels que le merveilleux et l'onirique (via la poésie ${ }^{6}$ ) ou même le suspense (via le storytelling ${ }^{7}$ ). Par ailleurs, elle n'hésite pas à exploiter et à se servir de toute une palette d'outils y compris numériques $^{8}$. Néanmoins, Louise Campbell n'idéalise pas ces premiers contacts et souligne les freins (ou obstacles) qu'elle rencontre couramment avec les participants. Là encore, son regard de médiatrice lui permet de les interpréter non pas comme des obstacles mais bien plutôt comme des leviers possibles, comme des points d'appuis pour ses activités.

L. c. : Par exemple, très souvent, les personnes à qui je m'adresse pensent que les musiciens et les compositeurs sont des gens qui n'ont rien à voir avec eux, que ce sont des personnes inaccessibles. Alors je parle de leur vécu, pour que chacun puisse réduire la distance mentale induite par la célébrité. J'aime beaucoup 1'histoire de la musique, je vais donc toujours raconter des petites histoires, pas comme un cours d'histoire mais plutôt sur le mode du "savez-vous que...? ", quelque chose d'un peu plus personnel, de plus proche. Je n'hésite pas à dire que ce sont des gens ordinaires comme toi et moi qui ont fait de la musique. Par exemple, je raconte 1'histoire de Brahms et du clarinettiste Richard Mühlfeld. Brahms, qui avait pris sa retraite, adorait

\footnotetext{
6 Un exemple d'activité de ce type réalisée par Louise Campbell : Poèmes sonores, http://louisecampbell.ca/wp/fr/2017/10/, consulté le 23 avril 2020.

7 Élaboration d'un récit à la manière d'un conte. Un exemple : Un bateau des Îles, http://louisecampbell.ca/wp/fr/2019/12/12/english-a-boat-saves-christmas/, consulté le 22 avril 2020.

8 Un exemple : Loops en folie, http://louisecampbell.ca/wp/fr/2018/06/, consulté le 22 avril 2020.
} 
tellement le jeu de Richard Mühlfeld qu'il est sorti de sa retraite pour écrire une pièce spécialement pour lui ! Il y a beaucoup de gens qui ne comprennent pas ça, qui ne connaissent pas ça, qui ne savent pas non plus qu'il y a eu plein de lettres échangées entre des compositeurs et des interprètes. Il y a vraiment des choses intéressantes à raconter aux gens. Ça les intéresse de découvrir des trajectoires humaines autant qu'artistiques.

\section{SE NOMMER MÉDIATRICE DE LA MUSIQUE}

Si la médiation de la musique occupe une grande part de l'activité professionnelle de Louise Campbell, elle-même ne se nomme comme telle que depuis quelques années. Créer des liens entre la musique et les publics était pour la médiatrice une pratique naturelle et intuitive. Récemment, les contours de cette pratique se sont peu à peu définis pour être finalement constitutifs d'une compétence qualifiée, organisée et répertoriée sous la terminologie de « médiation de la musique ».

F. B. : Peux-tu m'en dire davantage sur ton parcours, comment en es-tu venue à pratiquer la médiation de la musique?

L. c. : Ça, je pense que je l'ai toujours fait. Je crois ne jamais m'être posée la question de nommer ma pratique avant que tu ne me poses la question ! Quand j'ai lu la question [que tu me posais par courriel], je me suis dit: "OK, il faut que je me pose la question, là ! " Je pense que c'est venu naturellement avec ma première prof de musique - qui était aussi ma grand-mère, une musicienne qui faisait de la direction de chant choral. J'avais toujours besoin de présenter la musique avant de jouer. J'avais toujours besoin de faire une petite présentation, de me nommer moi-même, nommer le compositeur, nommer la pièce... peu importe si c'était moi qui l'avais composée ou quelqu'un d'autre. Donc je pense que, pour moi, ça a toujours été une évidence de me demander comment faire des liens avec les autres. Je le fais depuis que je suis toute petite. Puis je pense aussi qu'il y a une aptitude pour la musique, puis il y a une aptitude pour la communication et l'éducation. Et je pense que j'avais toujours cet intérêt en moi de transmettre. La médiation, c'est en partie de l'enseignement et la communication de la musique avec les autres. C'est vraiment cet intérêt-là que je porte, l'intérêt et la curiosité de voir comment les autres reçoivent la musique. Parce que finalement on peut seulement faire ça. On ne peut pas être dans les oreilles des autres, on peut seulement appréhender cela avec le partage de la musique, avec la discussion créée autour, ou en faisant de la musique avec les autres.

F. B. : Finalement, tu t'es formée toi-même à la médiation ? Tu n'as pas reçu de formation pour cela?

L. c. : Non, je n'ai jamais reçu de formation en médiation. Ça a toujours été quelque chose que j'ai fait moi-même. [...] J'ai une maîtrise en pédagogie, mais honnêtement, c'était plus orienté recherche que pratique de la médiation. En revanche, il y a beaucoup de profs dans ma famille et j'ai eu mes premiers emplois dans des camps d'été sportifs. Nous étions la plupart du temps à l'extérieur, en plein air. On faisait toujours des choses participatives, actives et je trouve qu'il y a beaucoup de similarités 
avec la musique. Donc, pour moi c'était une évidence de faire des activités participatives avec la contribution de tout le monde autour de moi. Il ne s'agit pas juste de parler avec du monde. Je dirais que c'est plus ça ma formation que quelque chose que j'ai appris à l'université. Ce sont les gens [les participants à mes activités] qui m'ont formée. Ce sont eux qui donnent les réponses et les possibilités, parfois totalement différemment de ce que j'aurais imaginé. Donc j'ai toujours fait de la médiation de la musique en réponse aux besoins des gens et en essayant de comprendre les spécificités des divers contextes dans lesquels je suis amenée à intervenir. Je l'ai fait même avant de connaître le terme de "médiation ", et avant même que ce terme-là ne recouvre une réalité avec ses théories, ses savoirs et savoir-faire. Ça, c'est venu vraiment juste ces dernières années, mais avant ça, c'était juste : " oK, j'ai besoin de faire de la musique avec ces gens qui sont devant moi. Comment est-ce que je vais m'y prendre? Comment est-ce qu'on peut faire de la musique ensemble? »

Ce témoignage de Louise Campbell semble parfaitement faire écho au mémoire de Pierre Jeannot ${ }^{9}$, qui, partant d'entretiens avec plusieurs acteurs du champ culturel français, fait apparaître que ces derniers exerçaient bien la médiation de la musique sans même le savoir ou avant que l'activité ne soit nommée comme telle par les institutions artistiques ou éducatives. Ces responsables de « département d'éducation » ou " animateurs culturels " justifiaient d'ailleurs leur incapacité à se définir comme " médiateur·trice » par le fait qu'ils n'aient pas reçu de formation diplômante dans ce domaine. À l'instar des médiateur·tice·s interrogé·e·s en 2018 par Irina Kirchberg ${ }^{10}$, ils expliquaient n'avoir saisi que récemment la signification et les savoirs théoriques associés à la pratique de cette discipline.

Définir ce qu'est la médiation de la musique est une tâche complexe, ce qui a présenté et présente toujours des freins à sa professionnalisation, car rien n'est encore figé. Cela peut s'expliquer ainsi : par définition, le médiateur est médian, c'est-à-dire transverse à une multitude de fonctions et services. Ce constat particulièrement criant est réalisé par les chercheurs qui se penchent sur les conditions de travail des médiateur·tice·s parfois pris·e·s, surtout dans les institutions culturelles, dans des configurations d'emploi les amenant à réaliser des tâches liées au marketing, à la communication, la production artistique, la programmation, et à l'éducation, etc. ${ }^{11}$. La fiche de poste d'un médiateur ne sera alors jamais exhaustive tant il lui est

9 Pierre Jeannot (2016), Médiation de la musique. Une profession ? Portraits de médiateurs de la musique classique, mémoire de Master, Université Paris-Sorbonne.

10 Irina Kirchberg (2018), Panorama de la médiation de la musique au Québec. Définitions, acteurs, enjeux, Montréal, Partenariat sur les Publics de la Musique $\left(\mathrm{P}^{2} \mathrm{M}\right) /$ Conseil Québécois de la Musique (CQM), https://p2m.oicrm.org/wp-content/uploads/2019/07/V2-KIRCHBERG-Panorama-de-la-me\%cc\%81diation-de-la-musique-au-Que\%cc\%81bec-FINAL-mars-2019-1.pdf, consulté le 22 avril 2020.

11 Voir : Nicolas Aubouin, Frédéric Kletz, et Olivier Lenay (2010), « Médiation culturelle. L’enjeu de la gestion des ressources humaines ", Culture études, vol. 1, no 1, p. 1-12, https://www.cairn.info/revueculture-etudes-2010-1-page-1.htm, consulté le 22 avril 2020 ; Bernadette Dufrêne et Michèle Gellereau (2003), "Qui sont les médiateurs culturels ? Statuts, rôles et constructions d'images ", MEI " Médiation et information ", $\mathrm{n}^{\circ}$ 19, p. 163-175, http://www.mei-info.com/wp-content/uploads/revue19/ilovepdf.com split 11.pdf, consulté le 22 avril 2020. 
nécessaire d'intervenir au sein d'un écosystème culturel complexe qui l'oblige à avoir des compétences polyvalentes - par exemple, être lui-même musicien - et surtout à savoir être en lien et mettre en lien des entités qui, pour beaucoup, n'ont pas des habitudes de travail collaboratif.

À considérer la société occidentale comme une mécanique hyper structurée et souvent très clivée - chacun est à une place donnée, avec son périmètre de compétences et de responsabilités -, on comprendra vite que si une proposition ou une personne n'est ni d'ici ni de là, alors elle n'est nulle part. La polyvalence de l'activité du médiateur, en tant que " tiers » non situé dans une identité exclusive, rend la définition de son activité difficile à cerner et sa fiche de poste complexe à établir. La reconnaissance de son activité en tant que profession demande alors un changement de perspective. Les choses semblent évoluer aujourd'hui au Québec et ce témoignage de Louise Campbell en est la preuve tout comme l'émergence de formations ${ }^{12}$ professionnalisantes de haut niveau universitaire et d'associations de médiateurs culturels ${ }^{13}$ qui, à l'instar du MéMuQ, se donnent pour mission « de promouvoir et favoriser une vie culturelle musicale active, dynamique et inclusive pour tou-te-s les québécois-e-s et de créer et alimenter un espace de partage et de convergence des ressources professionnelles pour les médiateurs et médiatrices de la musique au Québec ${ }^{14}{ }^{\text {». }}$

12 Les formations en médiation culturelle et/ou de la musique au Québec : le D.E.S.s. en médiation de la musique (Université de Montréal) et l'AEC - Spécialisation en médiation culturelle (cégep Saint-Laurent).

13 Associations québécoises de médiation culturelle et musicale en plein développement : le regroupement des médiateurs culturels du Québec ( $\mathrm{rMcQ}$ ), groupe Facebook.

14 LesmédiateursetmédiatricesdelamusiqueduQuébec(MéMuQ):https://memuq.org/?fbclid=IwAR1FLwepKLjWswQcMsurpFCmD Sj0vWn3PDaci9v01evaRUGLCDePk0Hwr4, consulté le 22 avril 2020. 